\title{
Iron-sparing Response of Mycobacterium avium subsp. paratuberculosis is strain dependent
}

Harish K Janagama', Senthilkumar ${ }^{1,6}$, John P Bannantine ${ }^{3}$, Abirami Kugadas' ${ }^{1}$ Pratik Jagtap ${ }^{4}$, LeeAnn Higgins ${ }^{5}$, Bruce A Witthuhn ${ }^{5}$, Srinand Sreevatsan ${ }^{1,2^{*}}$

\begin{abstract}
Background: Two genotypically and microbiologically distinct strains of Mycobacterium avium subsp. paratuberculosis (MAP) exist - S and C MAP strains that primarily infect sheep and cattle, respectively. Concentration of iron in the cultivation medium has been suggested as one contributing factor for the observed microbiologic differences. We recently demonstrated that $\mathrm{S}$ strains have defective iron storage systems, leading us to propose that these strains might experience iron toxicity when excess iron is provided in the medium. To test this hypothesis, we carried out transcriptional and proteomic profiling of these MAP strains under iron-replete or -deplete conditions.

Results: We first complemented M. smegmatis $\triangle i d e R$ with IdeR of C MAP or that derived from S MAP and compared their transcription profiles using M. smegmatis $m c^{2} 155$ microarrays. In the presence of iron, sldeR repressed expression of bfrA and MAP2073c, a ferritin domain containing protein suggesting that transcriptional control of iron storage may be defective in $\mathrm{S}$ strain. We next performed transcriptional and proteomic profiling of the two strain types of MAP under iron-deplete and -replete conditions. Under iron-replete conditions, C strain upregulated iron storage (BfrA), virulence associated (Esx-5 and antigen85 complex), and ribosomal proteins. In striking contrast, $\mathrm{S}$ strain downregulated these proteins under iron-replete conditions. iTRAQ (isobaric tag for relative and absolute quantitation) based protein quantitation resulted in the identification of four unannotated proteins. Two of these were upregulated by a C MAP strain in response to iron supplementation. The iron-sparing response to iron limitation was unique to the $C$ strain as evidenced by repression of non-essential iron utilization enzymes (aconitase and succinate dehydrogenase) and upregulation of proteins of essential function (iron transport, [Fe-S] cluster biogenesis and cell division).

Conclusions: Taken together, our study revealed that $C$ and $S$ strains of MAP utilize divergent metabolic pathways to accommodate in vitro iron stress. The knowledge of the metabolic pathways these divergent responses play a role in are important to 1) advance our ability to culture the two different strains of MAP efficiently, 2) aid in diagnosis and control of Johne's disease, and 3) advance our understanding of MAP virulence.
\end{abstract}

\section{Background}

Mycobacterium avium subsp. paratuberculosis (MAP), the causative agent of Johne's disease (JD) of ruminants, often requires eight to sixteen weeks to see colonies in culture - a major hurdle in the diagnosis and therefore in implementation of optimal control measures. Unlike other mycobacteria, which mobilize iron via mycobactins, MAP is unable to produce detectable mycobactin

\footnotetext{
* Correspondence: sreev001@umn.edu

'Department of Veterinary Population Medicine, University of Minnesota, Saint Paul MN, USA

Full list of author information is available at the end of the article
}

in vitro or in vivo [1-3]. Although the reasons for the in :vitro mycobactin dependency of MAP are currently unknown, we have recently shown that the mycobactin $(m b t)$ operon promoter is active and that the mycobactin genes are transcribed by MAP inside macrophages [4] and in tissues of naturally infected animals (accepted for publication in BMC Genomics).

Pathogenic mycobacteria encounter a wide variety of stressors inside the host cells and their ability to overcome iron deprivation and iron toxicity represents a major virulence determinant [5]. Transcript and protein profiling of MTB and other pathogens in response to
C Biomed Central

() 2010 Janagama et al; licensee BioMed Central Ltd. This is an Open Access article distributed under the terms of the Creative Commons Attribution License (http://creativecommons.org/licenses/by/2.0), which permits unrestricted use, distribution, and reproduction in any medium, provided the original work is properly cited. 
in vitro iron stress is well documented [6-9]. While MAP transcriptome or proteome profiles in response to heat shock, $\mathrm{pH}$, oxidative stress, hypoxia, and nutrient starvation have been demonstrated [10-12], stress responses to iron supplementation or starvation are lacking.

Iron dependent regulator (IdeR) has been very well studied as a global regulator involved in maintaining iron homeostasis in Mycobacterium tuberculosis (MTB) [13]. Recently we have demonstrated that IdeR of MAP in the presence of iron recognizes a consensus sequence on the promoter called "iron box" and regulates expression of genes involved in iron acquisition $(m b t)$ and storage $(b f r A)$. More interestingly, we demonstrated that polymorphisms in the promoter of iron storage gene $(b f r A)$ in $\mathrm{S}$ MAP strains relative to $\mathrm{C}$ MAP strains results in a differential gene regulation [4]. IdeR dependent repression of $b f r A$ in the presence of iron suggests variations in iron storage mechanisms and/or iron requirements in cattle and sheep MAP strains.

Comparative genomic hybridizations, short sequence repeat analysis and single nucleotide polymorphisms of MAP isolates obtained from diverse host species have established and indexed genomic differences between $\mathrm{C}$ and S strains of MAP [14-19]. Phylogenetic analysis of sequences has identified $\mathrm{C}$ and $\mathrm{S}$ strains as separate pathogenic clones that share a common ancestor [20-23]. Furthermore, cellular infection studies show distinctive phenotypes between the two MAP strain types $[24,25]$. We also recently demonstrated that S strains have defective iron storage systems, leading us to propose that these strains might experience iron toxicity when excess iron is provided in the medium [4]. Taken together, the literature suggests that MAP strains vary in their iron dependent gene regulation. To test this further, we profiled their transcriptomes and proteomes in response to iron and demonstrated that iron induced metabolic pathways are significantly diverse.

\section{Methods}

\section{Bacterial strains, DNA manipulations and media}

Mycobacterium avium subsp. paratuberculosis strains MAP1018 (C MAP) and MAP7565 (S MAP) were grown in Middlebrook 7H9 supplemented with OADC enrichment medium and mycobactin J $(2 \mathrm{mg} / \mathrm{mL}$; Allied Monitor, Fayette, MO).

To test the hypothesis that gene regulation may be dependent on iron availability MAP strains were grown in Middlebrook $7 \mathrm{H} 9$ medium without mycobactin $\mathrm{J}$ or Sauton medium $\left(0.5 \mathrm{~g} \mathrm{KH}_{2} \mathrm{PO}_{4}, 0.5 \mathrm{~g} \mathrm{MgSO}_{4}, 4.0 \mathrm{~g} \mathrm{L-}\right.$ asparagine, $60 \mathrm{ml}$ glycerol, $0.05 \mathrm{~g}$ ferric ammonium citrate, $2.0 \mathrm{~g}$ citric acid, $0.1 \mathrm{ml} 1 \%(\mathrm{w} / \mathrm{v}) \mathrm{ZnSO}_{4}$ and 2.5 $\mathrm{ml} 20 \%$ Tween 80 in 1 liter). Growth of MAP strains in the absence of mycobactin J took over 6 months to provide sufficient material for proteomics and transcriptional profiling. For iron restriction, 2,2'-dipyridyl (Sigma Aldrich, St. Louis, MO) was added at a concentration of $200 \mu \mathrm{M}$.

MAP7565 and MAP1018 have been genotyped by SSR as well as comparative genomics using oligoarrays. They represent the typical genomotypes of sheep and cattle strains, respectively [18] and show distinct phenotypes in both human and bovine macrophages [24,25].

$M$. smegmatis $\left(\mathrm{mc}^{2} 155\right)$ and $E$. coli TOP10F (Invitrogen Corporation, Carlsbad, CA) competent cells were grown in Luria Bertani (LB) medium and antibiotics (kanamycin $(20 \mu \mathrm{g} / \mathrm{ml})$ or hygromycin $(100 \mu \mathrm{g} / \mathrm{ml})$ ) were added when necessary. The open reading frames of ideR (MAP2827) derived from C or S MAP strains were cloned into pSM417 and M. smegmatis $\Delta i d e R$ (SM3) was complemented as previously reported [4]. Briefly, MAP2827 from MAP1018 (cideR) or MAP 7565 (sideR) was amplified via PCR using primers that carried restriction sites for BamHI and HindIII. Amplified products were double digested with BamHI and HindIII and ligated into a pre digested (BamHI and HindIII) expression plasmid pSM417. Accuracy of the ligation and orientation of MAP2827 in pSM417 was verified by sequencing. SM3 was transformed with pSM417 carrying MAP2827 from C or S MAP strains.

A seed stock from logarithmically grown $\left(\mathrm{OD}_{600}=\right.$ 1.0) cultures were diluted to fresh medium to yield an $\mathrm{OD}_{600}=0.1$. These were grown in various aliquots under constant shaking $(120 \mathrm{rpm})$ at $37^{\circ} \mathrm{C}$. These cultures were monitored for their growth at weekly intervals by measuring their absorbance at $600 \mathrm{~nm}$ wave length using SpectraMax M2 (Molecular Devices, Sunnyvale, CA) until they reached an absorbance of 1.0 (Additional file 1, Figure S1). At this point, the cultures were then pelleted, washed in ice cold 1 XPBS and resuspended in fresh culture medium (with or without the addition of 2,2'-dipyridyl (Sigma Aldrich, St. Louis, MO)). Dipyridyl was added at a concentration of 200 $\mu \mathrm{M}$. Following three hours of incubation at $37^{\circ} \mathrm{C}$ under constant shaking, cells were pelleted and washed with ice cold 1X PBS and either used in microarrays or iTRAQ. The detailed experimental design is provided as Additional file 1, Figure S2.

\section{Nucleic acid and protein extraction}

Log phase MAP or M. smegmatis cultures were pelleted, washed and re-suspended in fresh culture medium with or without $200 \mu \mathrm{M}$ of 2,2'-dipyridyl. The cultures were incubated at $37^{\circ} \mathrm{C}$ with shaking for $3 \mathrm{hr}$. immediately prior to RNA and protein extraction.

For RNA, cells were homogenized in Mini bead-beater for $4 \mathrm{~min}$. by adding $0.3 \mathrm{ml}$ of $0.1 \mathrm{~mm}$ sterile RNasefree zirconium beads followed by extraction using Trizol 
(Invitrogen, Carlsbad, CA). All samples were treated with RNase-free DNase I (Ambion, Inc., Austin, TX) to eliminate genomic DNA contamination. The purity and yield of total RNA samples was confirmed using Agilent 2100E Bioanalyzer (Agilent Technologies, Inc., Santa Clara, CA). RNA was stored at -80 until used in microarrays and real time RT-PCR assays.

For protein, cells were re-suspended in minimal quantity $(250 \mu \mathrm{L})$ of iTRAQ dissolution buffer (0.5 M TEAB $\mathrm{pH} 8.5$ ) and $0.1 \%$ SDS. The solution was transferred to a $2 \mathrm{ml}$ screw cap tube containing $0.1 \mathrm{~mm}$ zirconium beads (Biospec) and disrupted in minibead beater (Biospec) for $4 \times 1$ minute pulses with samples kept on ice between pulses. The lysate was then centrifuged at $12,000 \times \mathrm{g}$ for 10 minutes at $4^{\circ} \mathrm{C}$. Supernatant was transferred to a fresh tube without disturbing the pellet and used in iTRAQ labeling for detection of proteome (Additional file 1, Figure S3).

\section{Microarray experiments}

Gene expression profiling of S (1018) and C (7565) MAP strains was performed using MAP K-10 microarrays obtained from Dr. Michael Paustian, NADC, IA. Expression profiling of $M$. smegmatis $\Delta i d e R$ complemented with c or sideR was carried out using $M$. smegmatis $m c^{2} 155$ arrays provided via Pathogen Functional Genomics Resource Center (PFGRC) at J. Craig Venter Institute (JCVI). Array hybridizations and analyses were performed as described previously and according to the protocols established at PFGRC with minor modifications [26] and according to MIAME 2.0 guidelines.

Briefly, synthesis of fluorescently labeled cDNA (Cyanine-3 or Cyanine-5) from total RNA and hybridizations of labeled cDNA to MAP K-10 or $m c^{2} 155$ oligoarray was performed. Microarray hybridizations were performed from cDNA isolated from two independent experiments. On each independent occasion, bacterial cultures growing under iron-replete or iron-limiting medium were used for RNA extractions, cDNA labeling and array hybridizations. Each slide was competitively hybridized with cDNA obtained from iron-replete (labeled with cy3 or cy5) and iron-limiting growth medium (counter labeled with cy5 or cy3) to reveal relative expressional differences. About $4 \mu \mathrm{g}(2 \mu \mathrm{g}$ each from iron limitation or sufficient) of cDNA was used to hybridize onto the array. However, if the cDNA yield is low for a sample the RNA from the same sample was used to synthesize more cDNA, pooled and labeled onto the arrays. Hybridized slides were scanned using HP Scan array 5000 (PerkinElmer Inc., Waltham, MA). The images were processed and numerical data was extracted using the microarray image analysis software, BlueFuse (BlueGnome Ltd, Cambridge) and TM4 microarray suite available through JCVI. Genes differentially regulated at a fold change of 1.5 or greater were identified at a false discovery rate of $1 \%$ by Statistical Analysis of Microarrays (SAM) program [26]. Genes that showed a fold change 1.5 or greater in all the replicate arrays were retained and reported as being up- or downregulated in the presence of iron.

\section{Realtime RT-PCR}

RNA isolated from MAP strains grown under ironreplete or iron-limiting growth medium was used in real time RT-PCR assays. Genes were selected based on their diverse roles and microarray expression pattern. Selected genes included siderophore transport (MAP2413c, MAP2414c), esx-3 secretion system (MAP3783, MAP3784), aconitase (MAP1201c), fatty acid metabolism (MAP0150c) and virulence (MAP0216, MAP3531c, MAP1122 and MAP0475). RNA was treated with DNaseI (Ambion, Austin, TX) and one step Q-RT PCR was performed using QuantiFast SYBR Green mix (Qiagen, Valencia, CA) and gene specific primers (Additional file 1, Table S1) in a Lightcycler 480 (Roche, Indianapolis, IN).

\section{iTRAQ experiments}

Protein extracted from the two MAP strains grown in iron-replete or iron-limiting medium was used in iTRAQ analysis (Additional file 1, Figure S3). iTRAQ labeling and protein identification was carried out as described previously with minor modifications [27].

Briefly, cell lysate was quantified using the bicinchoninic acid (BCA) protein assay (Pierce, Rockford, IL) prior to trypsin digestion. Peptides were labeled with iTRAQ reagents (114 and 115 for MAP 1018 grown in ironreplete and iron-limiting medium respectively; 116 and 117 for MAP 7565 grown in iron-replete and iron-limiting medium respectively) at lysine and arginine amino terminal groups. The labeled peptides were pooled, dried and re-suspended in $0.2 \%$ formic acid. The re-suspended peptides were passed through Oasis ${ }^{\oplus}$ MCX 3CC $(60 \mathrm{mg})$ extraction cartridges per manufacturer recommendations (Waters Corporation, Milford, MA) for desalting prior to strong cation exchange (SCX) fractionation.

Eluted peptides were dried and dissolved in SCX buffer A $\left(20 \% \mathrm{v} / \mathrm{v} \mathrm{ACN}\right.$ and $5 \mathrm{mM} \mathrm{KH}_{2} \mathrm{PO}_{4} \mathrm{pH} 3.2$, with phosphoric acid) and fractionated using a polysulfoethyl A column $(150 \mathrm{~mm}$ length $\times 1.0 \mathrm{~mm}$ ID, $5 \mu \mathrm{m}$ particles, $300 \AA$ pore size) (PolyLC Inc., Columbia, MD) on a magic 2002 HPLC system (Michrom BioResources, Inc., Auburn, CA). Peptides were eluted by running a $0-20 \%$ buffer $\mathrm{B}$ gradient for greater than $55 \mathrm{~min}$. and $20 \%$ $100 \%$ buffer B $\left(20 \% \mathrm{v} / \mathrm{v} \mathrm{ACN}, 5 \mathrm{mM} \mathrm{KH_{2 }} \mathrm{PO}_{4} \mathrm{pH} 3.2\right.$, $500 \mathrm{mM} \mathrm{KCL}$ ) for $20 \mathrm{~min}$. at a column flow rate of 50 $\mu \mathrm{l} / \mathrm{min}$. Several fractions were collected at frequent 
intervals and seven fractions that showed mAU280 $>2$ were analyzed by LC-MS/MS as previously described [28].

Fractions were reconstituted in reversed-phase load buffer (10 $\mathrm{mM}$ phosphate buffer) and analyzed in a 4800 MALDI TOF/TOF instrument (AB Sciex, Foster City, CA). Protein pilot Software ${ }^{\mathrm{Tw}}$ 3.0.1 (AB Sciex, Foster city, CA) which utilizes the paragon ${ }^{\mathrm{mx}}$ scoring algorithm [29] was used to identify and quantify the relative abundance of the labeled peptides. Relative abundance of proteins (iron-replete $\mathrm{v} / \mathrm{s}$ iron-limitation) for each MAP strain was determined by comparing the reporter ion ratios (114/115 for $\mathrm{C}$ and $116 / 117$ for S MAP). iTRAQ experiments were repeated on two independent experiments for each treatment of each strain. We searched against the MAP K-10, non redundant (nr) mycobacteria proteins and entire $\mathrm{nr}$ protein database deposited in the NCBI along with the contaminants to identify MAP specific peptides at a false discovery rate of $1 \%$.

\section{Results}

\section{Transcriptional profiling of MAP IdeR}

We recently characterized MAP IdeR and computationally predicted that IdeR in the presence of iron regulates expression of 24 genes [4]. In the current study, we identified that 20 of the 24 previously predicted genes were differentially expressed in response to iron by MAP microarrays. Mycobactin synthesis, transport and fatty acid biosynthesis genes were repressed in the presence of iron by both cattle and sheep MAP strains (Additional file 1, Table S2). However iron storage and oxidoreductase genes were upregulated in the presence of iron only in C MAP (Additional file 1, Figure S4).

We first confirmed if these differences are due to regulation via IdeR. IdeR is essential in MAP and attempts to delete this gene failed [26]. We complemented $M$. smegmatis $\Delta$ ideR (SM3) with $\mathrm{C}$ or $\mathrm{S}$ strain $i d e R$ and compared regulational differences in the presence or absence of iron. Genes that showed a $\log _{2}$ fold change of 1.0 in SM3 or SM3 complemented with empty plasmid (negative controls) in the presence or absence of iron while having a fold change $> \pm 1.5$ in the complemented strains (test) and plasmid carrying $M$. smegmatis ideR and $m c^{2} 155$ (wild type) (positive control) were considered as being regulated by MAP IdeR. Fourteen of the 20 genes were regulated by IdeRs of both MAP strains in M. smegmatis. Furthermore, our results suggested that sIdeR functions by primarily repressing genes in the presence of iron whereas cIdeR functions both by repressing mycobactin synthesis and de-repressing iron storage genes in the presence of iron (Additional file 1, Table S3). These were further validated by realtime RT-PCR in both $M$. smegmatis transformants carrying MAP ideRs and MAP genetic background. The data is presented only for MAP (Additional file 1, Table S4).

We next compared the transcriptome and proteomes of $C$ and S MAP strains under iron-replete and ironlimiting conditions. Transcriptome analysis under two independent observations identified that 94 and 57 genes were consistently expressed by the $\mathrm{C}$ and $\mathrm{S}$ MAP strain respectively. Similarly, proteome data revealed a consistent expression of 64 and 60 proteins by the cattle and sheep MAP strains respectively. A comparison of these consistently detected transcripts and proteins revealed that, in the presence of iron, one third of the differentially regulated genes $(\mathrm{P}<0.05)$ were represented both in the respective transcriptome and the proteomes of the two strains (Figure 1).

\section{Transcript profiles under iron-limiting conditions}

Under iron-limiting conditions both the MAP strains showed increased transcription of genes belonging to mycobactin synthesis and esx-3, an essential secretory system of mycobactin biosynthesis (Additional file 1, Tables S2 - S5) [30].

$C$ MAP showed increased transcription of genes belonging to $\mathrm{ABC}$ type transporter proteins, suf operon involved in Fe-S cluster assembly proteins (MAP1187MAP1192), fatty acid biosynthesis operon (MAP3188MAP3190) and a pyruvate dehydrogenase operon (MAP2307c-MAP2309c) (Table 1 and Additional file 1, Table S5) suggesting that the transcriptional machinery is used to mobilize iron to maintain intracellular homeostasis. CMAP also upregulated expression of an enhanced intracellular survival gene (eis) (MAP2325), which was described as "deletion 3 " in sheep strains of MAP [16].

In contrast, the sheep strain of MAP in addition to upregulation of putative iron uptake and transport genes also expressed those belonging to heat shock proteins, molecular chaperones, and a VapBC family of toxinantitoxin operon (MAP2027c, MAP2028c) suggesting that iron deprivation might lead to a stringency response (Table 2 and Additional file 1, Table S6).

\section{Transcript profiles under iron-replete conditions}

There is increased protein synthesis and turnover in response to iron in M. tuberculosis (MTB) [31]. Similarly, the $\mathrm{C}$ strain upregulated as many as 25 rRNA genes, lipid metabolism, and several virulence-associated genes such as $f b p A$ (MAP0216) of antigen85 complex, soluble secreted antigen (MAP2942c), and oxidoreductase (MAP1084c) (Tables 3 and Additional file 1, Table S7). There was also an upregulation of MAP3296c, a whiB ortholog of $M$. tuberculosis that plays a role in antibiotic resistance and maintains intracellular redox 


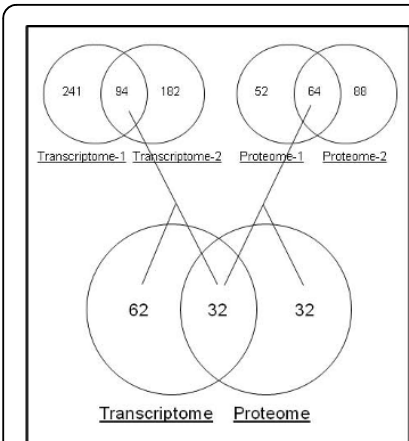

CATTLE MAP STRAIN

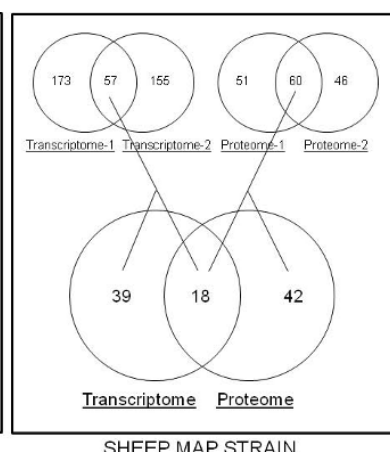

SHEEP MAP STRAIN
Figure 1 Transcriptome and proteome comparisons: Venn diagram showing the comparison of transcripts and proteins that were differentially expressed at a fold change of 1.5 or greater in cattle or sheep MAP strains in response to iron. One third of the genes differentially expressed in response to iron were represented in both the transcriptome and the proteome.

homeostasis [32]. Further, esx-5 operon which is present only in pathogenic mycobacteria and plays a role in cellcell migration of mycobacteria was upregulated [33]. A hypothetical protein (MAP0860c) upregulated in the presence of iron in the cattle strain of MAP has been described as a part of MAP-specific large sequence polymorphism (LSP4) [22].

In contrast, we did not document any upregulation (at a $\log _{2}$ fold change of 1.5) in the S MAP under ironreplete conditions. The directionality of transcripts as identified by microarrays under iron-replete conditions by $S$ MAP strain was confirmed by real time RT-PCR (Additional file 1, Table S4).

\section{Proteome}

The following criteria were used for protein identification in each treatment - (1) peptides identified by mass spectrometry were searched against the non-redundant (nr) protein database deposited in NCBI); and (2) MAP specific peptides reported with $>95 \%$ confidence were used to quantify the relative abundance (iron-replete v/s iron-limitation) of each protein. A peptide with no hits on the MAP genome but with identities with other mycobacterial proteins was considered as unannotated MAP protein.

\section{Protein expression under iron-limiting conditions}

Consistent with the transcription profile, the $\mathrm{C}$ strain of MAP upregulated proteins belonging to SUF operon involved in Fe-S cluster assembly, fatty acid metabolism and a pyruvate dehydrogenase (MAP2307c). Transporter proteins, two component systems, and cell division associated proteins (MAP1906c, MAP0448 and MAP2997c) were also upregulated by the $C$ strain (Table 1 and Additional file 1, Table S8). The sheep strain also upregulated transporter proteins, fatty acid biosynthesis, DNA replication protein (MAP3433), and stress response proteins (MAP3831c, MAP2764) (Table 2, Additional file 1, Table S9 and Figure S3).

Table 1 Transcript and protein expression in cattle MAP under iron-limiting (LI) conditions

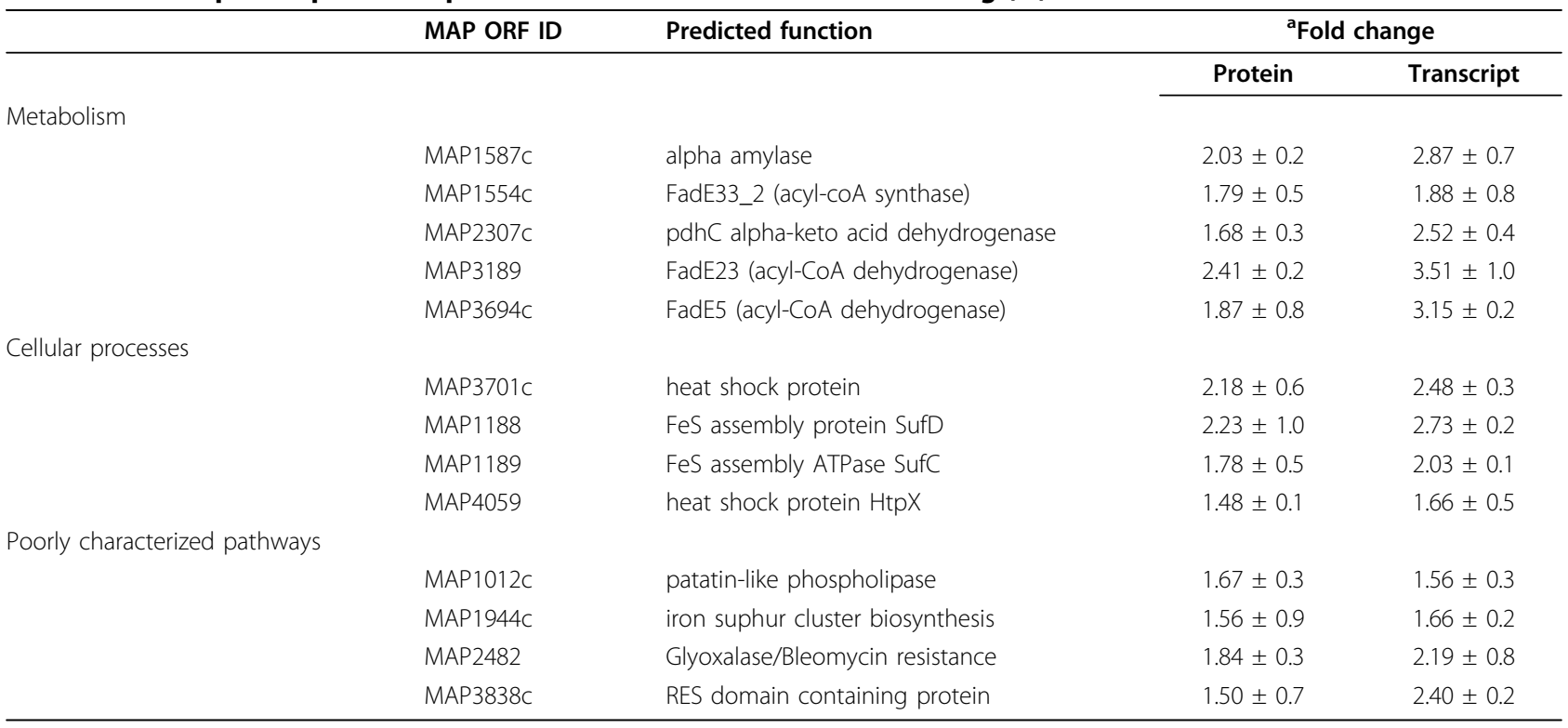

${ }^{a}$ MAP oligoarray was used to measure gene expression whereas iTRAQ was used to quantitate protein expression in the cultures of cattle MAP strain grown in iron-replete (HI) or iron-limiting (LI) medium. Fold change for each target was calculated and represented as a log $\mathrm{l}_{2}$ ratio of $\mathrm{LI} / \mathrm{HI}$. Shown are the MAP genes that demonstrated the presence of 1.5 times or more of transcripts and proteins in LI compared to HI. Genes are annotated based on the motif searches in KEGG database. 
Table 2 Transcript and protein expression in sheep MAP under iron-limiting (LI) conditions

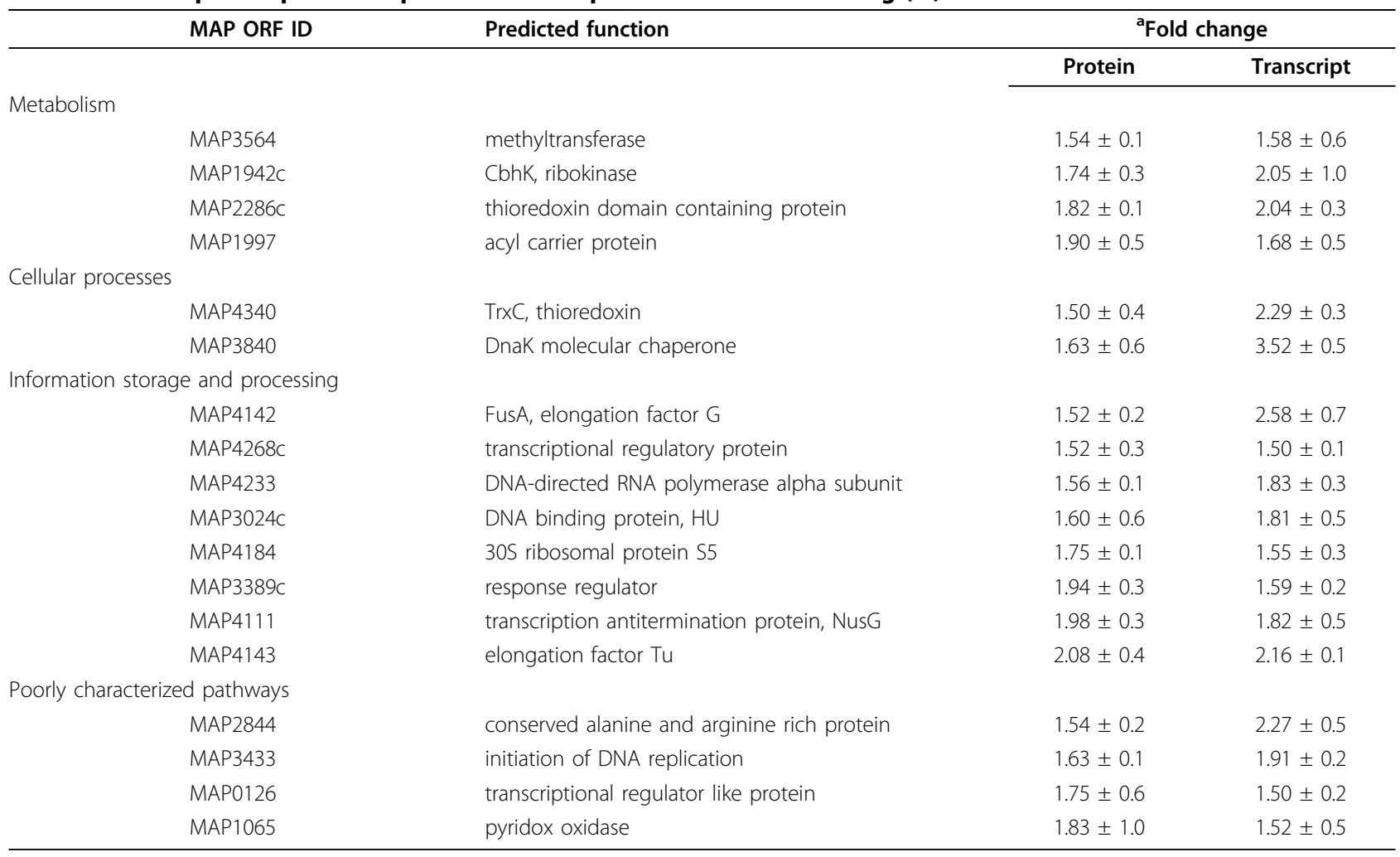

${ }^{a}$ MAP oligoarray was used to measure gene expression whereas iTRAQ was used to quantitate protein expression in the cultures of sheep MAP strain grown in iron-replete (HI) or iron-limiting (LI) medium. Fold change for each target was calculated and represented as a log $\mathrm{g}_{2}$ ratio of $\mathrm{LI} / \mathrm{HI}$. Shown are the MAP genes that demonstrated the presence of 1.5 times or more of transcripts and proteins in LI compared to HI. Genes are annotated based on the motif searches in KEGG database.

The iron-sparing response to iron starvation occurs when non-essential iron utilization proteins such as aconitase and succinate dehydrogenases are repressed and intracellular iron is used to maintain essential cellular functions $[34,35]$. Interestingly, during iron limitation, the cattle strain but not sheep MAP downregulated expression of aconitase (MAP1201c) and succinate dehydrogenases (MAP3697c, MAP3698c) (Figure 2). Repression of aconitase in response to iron starvation is post-transcriptionally mediated via small RNAs [36]. Consistent with this finding, our results reveal an upregulation of aconitase transcripts (both by microarray and Q-RT PCR) with a concomitant downregulation at the protein level in the C MAP alone under iron-limiting conditions.

\section{Protein expression under iron-replete conditions}

The sheep strain upregulated as many as 13 unique peptides ( $>95 \%$ confidence) that were mapped to MAP2121c. A representative peptide map is shown in Figure 3A. Interestingly, none of these were differentially regulated in response to iron by $\mathrm{C}$ strain of MAP. MAP2121c was originally described as $35-\mathrm{kDa}$ antigen and is an immune-dominant protein involved in MAP entry into bovine epithelial cells $[37,38]$. Although statistically not significant, further microarray analysis revealed a two-fold increase of MAP2121c in both cattle and sheep strains under iron-replete conditions (data not shown) suggesting a possible post transcriptional repression of MAP2121c by the cattle strain of MAP.

As expected, transcripts identified as upregulated under iron-replete conditions in C MAP strain were also upregulated in the proteome (Table 3, Additional file 1, Table S10). There was increased expression of five ribosomal proteins and a ribosome releasing factor (MAP2945c) by cattle MAP under iron-replete conditions. As previously reported, BfrA was upregulated in cattle MAP (Figure 3B). Antigen 85A and MAP0467c (mycobacterial heme, utilization and degrader) were also upregulated. However, MAP0467c and other stress response proteins were downregulated in the S MAP strain (Figure 4).

\section{Identification of unannotated MAP proteins}

We identified two unique peptides (SSHTPDSPGQQPPKPTPAGK and TPAPAKEPAIGFTR) that originated from the unannotated MAP gene located between MAP0270 (fadE36) and MAP0271 
Table 3 Transcript and protein expression in cattle MAP under iron-replete $(\mathrm{HI})$ conditions

\begin{tabular}{|c|c|c|c|}
\hline \multirow{2}{*}{$\begin{array}{l}\text { MAP ORF } \\
\text { ID }\end{array}$} & \multirow[t]{2}{*}{ Predicted function } & \multicolumn{2}{|c|}{${ }^{\mathrm{a}}$ Fold change } \\
\hline & & Protein & Transcript \\
\hline \multicolumn{4}{|l|}{ Metabolism } \\
\hline MAP0150c & $\begin{array}{l}\text { FadE25_2 (acyl-coA } \\
\text { dehydrogenase) }\end{array}$ & $1.72 \pm 0.1$ & $1.88 \pm 0.2$ \\
\hline MAP0789 & acetyl-CoA acetyltransferase & $1.73 \pm 0.3$ & $1.56 \pm 0.1$ \\
\hline MAP1846c & ATP phosphoribosyltransferase & $1.69 \pm 0.2$ & $3.68 \pm 0.3$ \\
\hline MAP2332C & Fas (fatty acid synthase) & $1.61 \pm 0.5$ & $2.28 \pm 0.4$ \\
\hline MAP3404 & $\begin{array}{l}\text { AccA3 (acetyl-/propionyl- } \\
\text { coenzyme A) }\end{array}$ & $1.45 \pm 0.1$ & $2.18 \pm 0.2$ \\
\hline MAP3698c & succinate dehydrogenase & $1.89 \pm 0.3$ & $4.57 \pm 0.5$ \\
\hline \multicolumn{4}{|c|}{ Cellular processes } \\
\hline MAP1339 & $\begin{array}{l}\text { iron regulated conserved } \\
\text { protein }\end{array}$ & $1.62 \pm 0.2$ & $0.78 \pm 0.3$ \\
\hline MAP1653 & thiol peroxidase & $1.79 \pm 0.5$ & $2.29 \pm 0.2$ \\
\hline \multicolumn{4}{|c|}{ Information storage and processing } \\
\hline MAP2907c & translation initiation factor IF-2 & $1.57 \pm 0.2$ & $1.89 \pm 0.2$ \\
\hline MAP2945c & ribosome releasing factor & $1.66 \pm 0.3$ & $2.11 \pm 0.5$ \\
\hline MAP4113 & 50 S ribosomal protein L1 & $1.61 \pm 0.1$ & $1.57 \pm 0.2$ \\
\hline MAP4125 & rplJ 50 S ribosomal protein L10 & $1.52 \pm 0.1$ & $1.66 \pm 0.5$ \\
\hline MAP4142 & fusA elongation factor $G$ & $2.13 \pm 0.4$ & $3.05 \pm 0.3$ \\
\hline MAP4160 & rps」 $30 \mathrm{~S}$ ribosomal protein $\mathrm{S10}$ & $1.68 \pm 0.3$ & $2.87 \pm 0.4$ \\
\hline MAP4181 & rpsH 30 S ribosomal protein $\mathrm{S} 8$ & $1.79 \pm 0.5$ & $2.42 \pm 0.1$ \\
\hline MAP4233 & $\begin{array}{l}\text { rpoA DNA-directed RNA } \\
\text { polymerase }\end{array}$ & $1.56 \pm 0.1$ & $1.65 \pm 0.4$ \\
\hline \multicolumn{4}{|c|}{ Poorly characterized pathways } \\
\hline MAP0216 & FbpA antigen 85-A & $1.87 \pm 0.2$ & $2.16 \pm 0.3$ \\
\hline MAP1122 & $\begin{array}{l}\text { mycobacterial integration host } \\
\text { factor }\end{array}$ & $1.73 \pm 0.3$ & $2.00 \pm 0.5$ \\
\hline
\end{tabular}

${ }^{a}$ MAP oligoarray was used to measure gene expression whereas ITRAQ was used to quantitate protein expression in the cultures of cattle MAP strain grown in iron-replete $(\mathrm{HI})$ or iron-limiting (LI) medium. Fold change for each target was calculated and represented as a $\log _{2}$ ratio of $\mathrm{HI} / \mathrm{LI}$. Shown are the MAP genes that demonstrated the presence of 1.5 times or more of transcripts and proteins in $\mathrm{HI}$ compared to LI. Genes are annotated based on the motif searches in KEGG database.

(ABC type transporter). We also identified two peptides (DAVELPFLHK and EYALRPPK) that did not map to any of the annotated MAP proteins but to the amino acid sequence of MAV_2400. Further examination of the MAP genome revealed that the peptides map to the reversed aminoacid sequence of MAP1839. These two unique proteins were not differentially regulated in response to iron. However, two more unique peptides that were translated from other unannotated MAP genes were upregulated ( $>1.5$ fold) under iron-replete conditions in C MAP strain (Figure 3B).

\section{Discussion}

Johne's disease is a major animal health problem of ruminant species worldwide and imposes significant economic losses to the industry. Our ability to culture

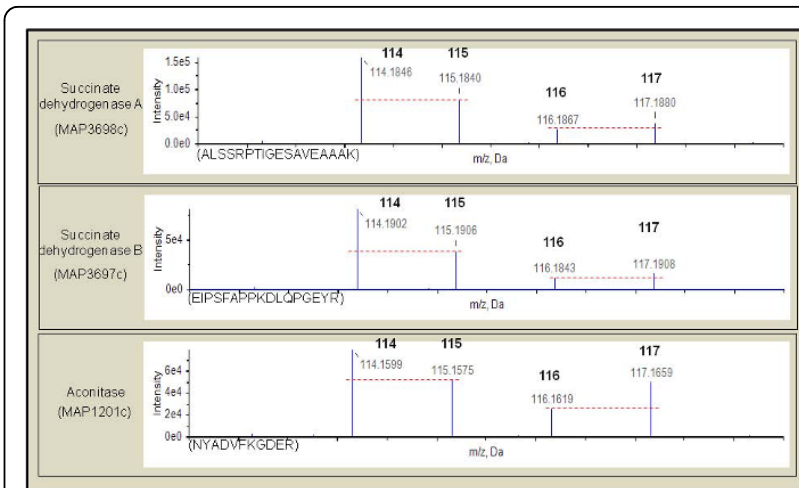

Figure 2 Repression of non-essential iron using proteins under iron-limiting conditions by cattle MAP strain: Reporter ion regions $(114-117 \mathrm{~m} / \mathrm{z})$ of peptide tandem mass spectrum from iTRAQ labeled peptides from MAP3698c, MAP3697c and MAP1201c are shown. Quantitation of peptides and inferred proteins are made from relative peak areas of reporter ions. Peptides obtained from cattle MAP cultures grown in iron-replete and iron-limiting medium were labeled with 114 and 115 reporter ions, respectively.. Peptides obtained from sheep MAP cultures grown in iron-replete and ironlimiting medium were labeled with 116 and 117 reporter ions, respectively. The peptide sequences and shown in the parenthesis and the red dashed line illustrates the reporter ion relative peak intensities. Cattle strain of MAP shows an iron sparing response by downregulating expression of iron using proteins.

the causative agent-Mycobacterium avium subsp. paratuberculosis (MAP)-and therefore its rapid diagnosis and our understanding of its virulence is limited. MAP is difficult to culture because of its unusually strict iron requirements. For optimal growth in laboratory media, MAP requires a siderophore (mycobactin) supplementation that makes MAP fastidious [39]., often requiring eight to sixteen weeks to produce colonies in culture - a major hurdle in the diagnosis and therefore implementation of optimal control measures. Understanding iron regulatory networks in the pathogen invitro is therefore of great importance.

\section{A tale of two strain types of MAP - A case to study iron regulation}

Several microbiological and genotyping studies and clinical observations suggest that Johne's in certain hosts such as sheep, goats, deer, and bison is caused by a distinct set of strains that show a relatively high degree of host preference $[18,40]$. At least two microbiologically distinct types of MAP have been recognized. A less readily cultivable type is the common, but not invariable, cause of paratuberculosis in sheep (S MAP) $[39,41,42]$, while another readily cultivable type is the most common cause of the disease in cattle (C MAP). Cell infection studies have also revealed distinctive host response phenotypes between cattle and sheep MAP strains - the former elicit primarily a pro-inflammatory 


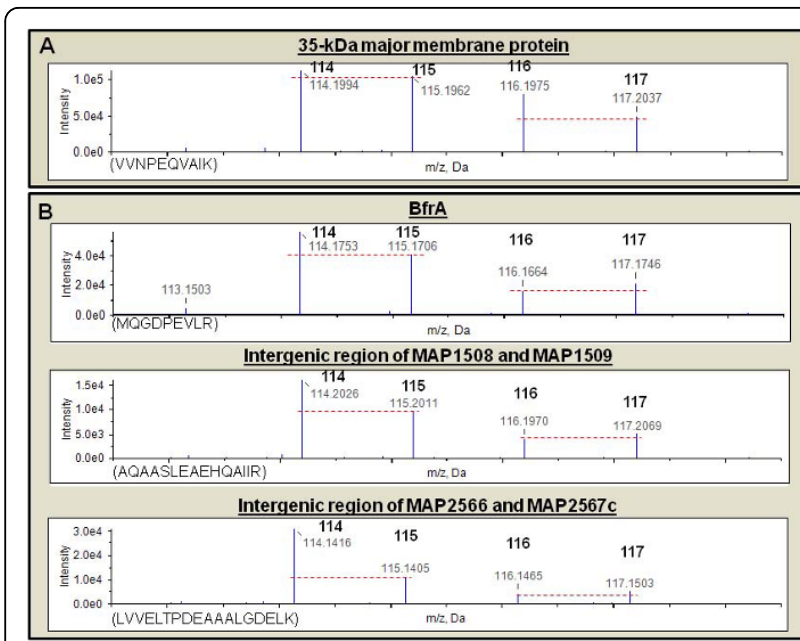

Figure 3 Peptide quantitation of proteins expressed by $C$ and S MAP strains under iron-replete conditions: Reporter ion regions $(114-117 \mathrm{~m} / \mathrm{z}$ ) of peptide tandem mass spectrum from iTRAQ labeled peptides from the (A) 35-kDa major membrane protein (MAP2121c) and (B) BfrA, and the intergenic regions of MAP1508-1509 and MAP2566-2567c. Quantitation of peptides and inferred proteins are made from relative peak areas of reporter ions. Several unique peptides (>95\% confidence) were mapped to each protein. However, only one representative peptide is shown for each protein. Peptides obtained from cattle MAP cultures grown in iron-replete and iron-limiting medium were labeled with 114 and 115 reporter ions, respectively. Peptides obtained from sheep MAP cultures grown in iron-replete and iron-limiting medium were labeled with 116 and 117 reporter ions, respectively. The peptide sequences and shown in the parenthesis and the red dashed line illustrates the reporter ion relative peak intensities. MAP2121c alone was upregulated in the sheep MAP strain under iron-replete conditions.

response while latter strains suppress inflammation and upregulate anti-apoptotic pathways [24,25]. In addition, since MAP genome sequence was published in 2005, very little research has focused on iron physiology and its contribution to metabolic networks of this fastidious organism.

Based on these classical microbiologic, genotypic, and clinical observations, we addressed the hypothesis that the iron dependent gene regulation is different between cattle and sheep MAP strains using a systems approach.

\section{Iron-sparing response to iron-limitation is unique to cattle MAP strain}

Iron is a critical component of several metabolic enzymes [43]. Most bacteria respond to iron starvation with a unique regulatory mechanism called the ironsparing response [35]. Iron-sparing is a physiological phenomenon used by cells to increase the intracellular iron pool by post-transcriptionally repressing the synthesis of non-essential iron using proteins and sparing iron for essential cellular functions [44]. Therefore, the

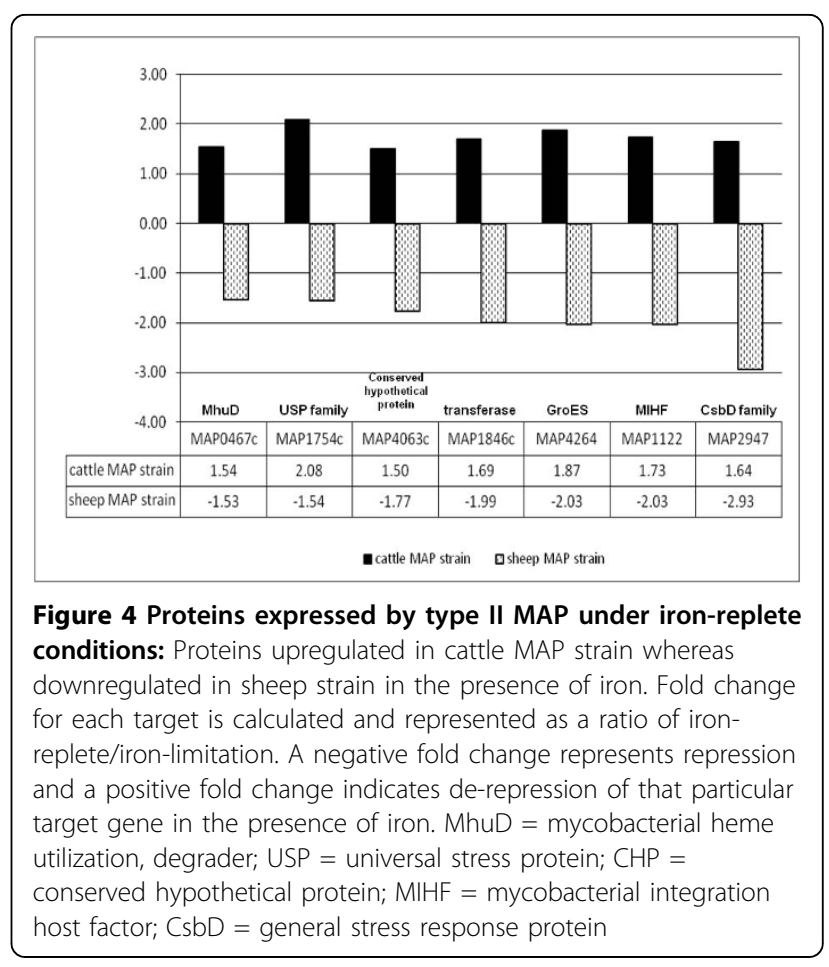

paradigm is to transcriptionally upregulate all iron uptake systems while repressing non-essential enzymes via post-transcriptional regulatory mechanisms to survive iron-limiting conditions. Both MAP strains upregulated genes involved in siderophore biosynthesis $(m b t)$, ability to acquire iron from synthesized siderophores $(e s x-3)$, and to transport iron bound siderophores (irtAB) into the bacterium (Additional file 1, Table S2). Furthermore, cattle MAP strain under iron-limiting conditions upregulated transcription of aconitase (Additional file 1, Table S4) while downregulating its protein expression (Figure 2). It is likely that targets for posttranscriptional repression of these non-essential iron using proteins are mediated via small RNAs [34]. Studies to test this hypothesis in the two MAP strain types are underway.

\section{Differential metabolic responses of cattle and sheep MAP strains to iron-limitation}

Under iron-limiting conditions most other bacteria including M. tuberculosis (MTB) upregulate SUF operon $[26,45]$. SUF synthesizes [Fe-S] clusters and transports them to iron-sulfur containing proteins involved in diverse cellular functions such as redox balance and gene regulation [46]. This is critical because unlike $E$. coli, MTB and MAP genomes encode for only one such system to synthesize all the [Fe-S] needed by the cell and free iron and sulfide atoms are toxic to cells [47]. Our data revealed that cattle strain, but not $\mathrm{S}$ strain 


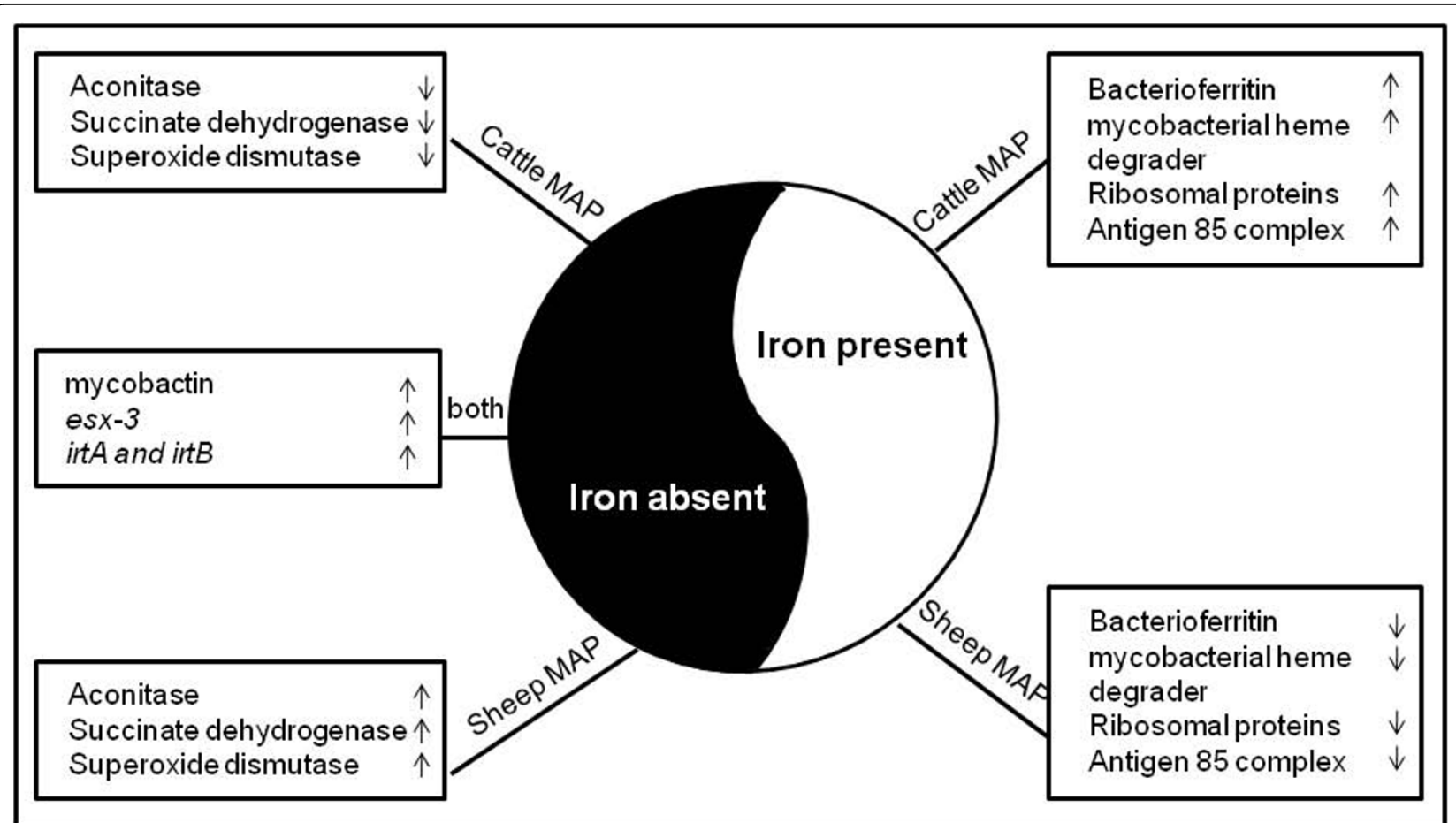

Figure 5 Iron dependent metabolic programming in cattle and sheep MAP: Under iron-replete conditions, there is upregulation of ribosomal proteins, bacterioferritin, mycobacterial heme, utilization and degrader proteins in cattle strain alone. Under iron limiting conditions, siderophore synthesis and transport genes are upregulated in both cattlel and sheep MAP strains. However, under iron limitation there is downregulation of aconitase, succinate dehydrogenases and superoxide dismutase in cattle MAP strain alone. This suggests an iron-sparing response exclusively in cattle but not sheep strain.

upregulated SUF operon at the transcript and protein level under iron-limiting conditions (Table 1).

Cattle MAP strain upregulated pyruvate dehydrogenase operon involved in catabolism of propionate a key component of lipid biosynthesis under limiting iron conditions [48]. In contrast, sheep strain upregulated isoprenoid synthesis genes involved in cell wall biogenesis [49]. The sheep isolate also upregulated oxidoreductase and stress responses in its transcriptome and proteome during iron-limitation (Table 2). CarD and toxin-antitoxin systems primarily function during unfavorable conditions such as starvation or oxidative stress by arresting cell growth $[50,51]$. Sheep strain upregulated transcripts of toxin-antitoxin system involved in arresting cell growth, suggesting a trend toward stringency response (Additional file 1, Table S6). Taken together, our data suggests that cattle strain is able to efficiently modulate its metabolism during iron-limitation - probably a survival advantage.

MAP2325, a hypothetical protein deleted in the sheep strain was found to be upregulated under iron-limiting conditions by the $\mathrm{C}$ strain (Additional file 1, Table S5). This is interesting because an ortholog of MAP2325 in MTB called enhanced intracellular survival (eis) interacts with host $\mathrm{T}$ cells. Stimulation of recombinant Eis from MTB results in increased production of IL-10 and decreased production of TNF- $\alpha$ thus contributing to mycobacterial survival inside macrophages [52]. We have also demonstrated a similar result in bovine or human macrophages stimulated with diverse MAP strains. Cattle strains produced relatively more IL-10 and less TNF- $\alpha$ and persisted for longer periods of time inside macrophages [24,25].

There is increased protein synthesis and turn over in response to iron in MTB [31]. Similarly, we observed an increased expression of ribosomal proteins in the transcriptome and proteome in C MAP under iron-replete conditions. In striking contrast, iron-limitation induced a similar theme in sheep strain. Heme degradation is a significant physiological phenomenon where in pathogens recycle iron and gain a survival advantage inside the host [53]. Recently the crystal structure of Rv3592 of MTB was solved and demonstrated its ability as heme degrader [54]. We observed an upregulation of MAP0467c protein (ortholog of Rv3592) under ironreplete conditions in C MAP while it was downregulated in the sheep strain (Figure 4). Similar to our previous reports, iron storage protein, BfrA was upregulated only 
by C MAP under iron-repletion (Figure 3) [4]. Although the reasons for differential iron storage mechanisms in sheep compared to cattle strains of MAP are currently unknown, differential role of ferritins in bacterial pathogens is not uncommon [55].

\section{Conclusions}

Our data revealed striking differences in metabolic pathways used by cattle and sheep strains of MAP to adapt to iron starvation (Figure 5). We have identified and characterized key iron dependent pathways of MAP. Since iron metabolism is critical for the invivo and invitro survival of the bacterium, our current studies are expected to improve our ability to provide better invitro culture methods for MAP and provide an understanding of iron regulation as a key virulence determinant of MAP.

\section{Additional material}

Additional file 1: Descriptive and pathway analysis of transcriptome and proteome data. This file contains the experimental design, additional microarray, proteomic and Q-RT PCR data along with pathway analysis of iron stress response proteins of $C$ and S MAP strains.

\section{Acknowledgements}

This work was supported in part by a USDA-NRI grant (2005-35204-16106) and Johne's disease Integrated Program (USDA-CSREES 2008-55620-18710) awarded to SS. We would like to thank Microbial and Plant Genomics Institute, Biomedical Genomics Center and Computational Genetics Laboratory at the University of Minnesota for providing resources and services to perform these studies. We would also like to thank JCVI for providing M. smegmatis microarrays.

\section{Author details \\ 'Department of Veterinary Population Medicine, University of Minnesota, Saint Paul MN, USA. ${ }^{2}$ Department of Veterinary and Biomedical Sciences, University of Minnesota, Saint Paul MN, USA. ${ }^{3}$ National Animal Disease Center, Agricultural Research Service, United States Department of Agriculture, Ames, IA, USA. ${ }^{4}$ Minnesota Supercomputing Institute for Advanced Computational Research, University of Minnesota, Minneapolis MN, USA. ${ }^{5}$ Department of Biochemistry, Molecular Biology and Biophysics, University of Minnesota, Minneapolis MN, USA. ${ }^{6}$ Department of Animal Biotechnology, Madras Veterinary College, Chennai, India.}

\section{Authors' contributions}

SS designed the study. HKJ participated in the experimental design with SS and performed most of the experiments. SK and AK helped in some experiments. JBP contributed to new reagents. BAW performed mass spectrometry. PJ and LAH helped in ITRAQ data analysis. HKJ and SS analyzed the data and wrote the manuscript. All authors read and approved the manuscript.

Received: 23 May 2010 Accepted: 22 October 2010 Published: 22 October 2010

\section{References}

1. Lambrecht RS, Collins MT: Mycobacterium paratuberculosis. Factors that influence mycobactin dependence. Diagn Microbiol Infect Dis 1992, 15(3):239-246.
2. Lambrecht RS, Collins MT: Inability to detect mycobactin in mycobacteriainfected tissues suggests an alternative iron acquisition mechanism by mycobacteria in vivo. Microb Pathog 1993, 14(3):229-238.

3. Snow GA: Mycobactins: iron-chelating growth factors from mycobacteria. Bacteriol Rev 1970, 34(2):99-125.

4. Janagama HK, Senthilkumar TM, Bannantine JP, Rodriguez GM, Smith I, Paustian ML, McGarvey JA, Sreevatsan S: Identification and functional characterization of the iron-dependent regulator (IdeR) of Mycobacterium avium subsp. paratuberculosis. Microbiology 2009, 155(Pt 11):3683-3690

5. Waddell SJ, Butcher PD: Microarray analysis of whole genome expression of intracellular Mycobacterium tuberculosis. Curr Mol Med 2007, 7(3):287-296.

6. Rao PK, Li Q: Protein turnover in mycobacterial proteomics. Molecules 2009, 14(9):3237-3258.

7. Rao PK, Roxas BA, Li Q: Determination of global protein turnover in stressed mycobacterium cells using hybrid-linear ion trap-fourier transform mass spectrometry. Anal Chem 2008, 80(2):396-406.

8. Rao PK, Li Q: Principal Component Analysis of Proteome Dynamics in Iron-starved Mycobacterium Tuberculosis. J Proteomics Bioinform 2009, 2(1):19-31.

9. Hindre $T$, Bruggemann $H$, Buchrieser $C$, Hechard $Y$ : Transcriptional profiling of Legionella pneumophila biofilm cells and the influence of iron on biofilm formation. Microbiology 2008, 154(Pt 1):30-41.

10. Gumber S, Whittington RJ: Analysis of the growth pattern, survival and proteome of Mycobacteriumavium subsp. paratuberculosis following exposure to heat. Vet Microbiol 2009, 136(1-2):82-90.

11. Gumber S, Taylor DL, Marsh IB, Whittington RJ: Growth pattern and partial proteome of Mycobacterium avium subsp. paratuberculosis during the stress response to hypoxia and nutrient starvation. Vet Microbiol 2009, 133(4):344-357.

12. Wu CW, Schmoller SK, Shin SJ, Talaat AM: Defining the stressome of Mycobacterium avium subsp. paratuberculosis in vitro and in naturally infected cows. J Bacteriol 2007, 189(21):7877-7886.

13. Rodriguez GM: Control of iron metabolism in Mycobacterium tuberculosis. Trends Microbiol 2006, 14(7):320-327.

14. Motiwala AS, Strother M, Amonsin A, Byrum B, Naser SA, Stabel JR, Shulaw WP, Bannantine JP, Kapur V, Sreevatsan S: Molecular epidemiology of Mycobacterium avium subsp. paratuberculosis: evidence for limited strain diversity, strain sharing, and identification of unique targets for diagnosis. J Clin Microbiol 2003, 41(5):2015-2026.

15. Motiwala AS, Strother M, Theus NE, Stich RW, Byrum B, Shulaw WP, Kapur V, Sreevatsan S: Rapid detection and typing of strains of Mycobacterium avium subsp. paratuberculosis from broth cultures. J Clin Microbiol 2005, 43(5):2111-2117

16. Marsh IB, Bannantine JP, Paustian ML, Tizard ML, Kapur V, Whittington RJ: Genomic comparison of Mycobacterium avium subsp. paratuberculosis sheep and cattle strains by microarray hybridization. J Bacteriol 2006, 188(6):2290-2293.

17. Marsh IB, Whittington RJ: Genomic diversity in Mycobacterium avium: single nucleotide polymorphisms between the $S$ and $C$ strains of $M$. avium subsp. paratuberculosis and with M. a. avium. Mol Cell Probes 2007, 21(1):66-75.

18. Paustian ML, Zhu X, Sreevatsan S, Robbe-Austerman S, Kapur V, Bannantine JP: Comparative genomic analysis of Mycobacterium avium subspecies obtained from multiple host species. BMC Genomics 2008, 9:135

19. Paustian ML, Kapur V, Bannantine JP: Comparative genomic hybridizations reveal genetic regions within the Mycobacterium avium complex that are divergent from Mycobacterium avium subsp. paratuberculosis isolates. J Bacteriol 2005, 187(7):2406-2415.

20. Turenne CY, Collins DM, Alexander DC, Behr MA: Mycobacterium avium subsp. paratuberculosis and M. avium subsp. avium are independently evolved pathogenic clones of a much broader group of M. avium organisms. J Bacteriol 2008, 190(7):2479-2487.

21. Turenne CY, Semret M, Cousins DV, Collins DM, Behr MA: Sequencing of hsp65 distinguishes among subsets of the Mycobacterium avium complex. J Clin Microbiol 2006, 44(2):433-440.

22. Alexander DC, Turenne CY, Behr MA: Insertion and deletion events that define the pathogen Mycobacterium avium subsp. paratuberculosis. J Bacteriol 2009, 191(3):1018-1025. 
23. Wu CW, Glasner J, Collins M, Naser S, Talaat AM: Whole-genome plasticity among Mycobacterium avium subspecies: insights from comparative genomic hybridizations. J Bacteriol 2006, 188(2):711-723.

24. Motiwala AS, Janagama HK, Paustian ML, Zhu X, Bannantine JP, Kapur V, Sreevatsan S: Comparative transcriptional analysis of human macrophages exposed to animal and human isolates of Mycobacterium avium subspecies paratuberculosis with diverse genotypes. Infect Immun 2006, 74(11):6046-6056

25. Janagama HK, Jeong K, Kapur V, Coussens P, Sreevatsan S: Cytokine responses of bovine macrophages to diverse clinical Mycobacterium avium subspecies paratuberculosis strains. BMC Microbiol 2006, 6:10.

26. Rodriguez GM, Voskuil MI, Gold B, Schoolnik GK, Smith I: ideR, An essential gene in mycobacterium tuberculosis: role of IdeR in iron-dependent gene expression, iron metabolism, and oxidative stress response. Infect Immun 2002, 70(7):3371-3381.

27. Seth M, Lamont EA, Janagama HK, Widdel A, Vulchanova L, Stabel JR, Waters WR, Palmer MV, Sreevatsan S: Biomarker discovery in subclinical mycobacterial infections of cattle. PLoS One 2009, 4(5):e5478.

28. Akkina SK, Zhang Y, Nelsestuen GL, Oetting WS, Ibrahlm HN: Temporal stability of the urinary proteome after kidney transplant: more sensitive than protein composition? J Proteome Res 2009, 8(1):94-103.

29. Shilov IV, Seymour SL, Patel AA, Loboda A, Tang WH, Keating SP, Hunter CL, Nuwaysir LM, Schaeffer DA: The Paragon Algorithm, a next generation search engine that uses sequence temperature values and feature probabilities to identify peptides from tandem mass spectra. Mol Cell Proteomics 2007, 6(9):1638-1655.

30. Siegrist MS, Unnikrishnan M, McConnell MJ, Borowsky M, Cheng TY, Siddiqi N, Fortune SM, Moody DB, Rubin EJ: Mycobacterial Esx-3 is required for mycobactin-mediated iron acquisition. Proc Natl Acad Sci USA 2009, 106(44):18792-18797.

31. Rao PK, Rodriguez GM, Smith I, Li Q: Protein dynamics in iron-starved Mycobacterium tuberculosis revealed by turnover and abundance measurement using hybrid-linear ion trap-Fourier transform mass spectrometry. Anal Chem 2008, 80(18):6860-6869.

32. Singh A, Guidry L, Narasimhulu KV, Mai D, Trombley J, Redding KE, Giles Gl, Lancaster JR Jr, Steyn AJ: Mycobacterium tuberculosis WhiB3 responds to $\mathrm{O} 2$ and nitric oxide via its [4Fe-4S] cluster and is essential for nutrient starvation survival. Proc Natl Acad Sci USA 2007, 104(28):11562-11567.

33. Abdallah AM, Verboom T, Hannes F, Safi M, Strong M, Eisenberg D, Musters RJ, Vandenbroucke-Grauls CM, Appelmelk BJ, Luirink J, et al: A specific secretion system mediates PPE41 transport in pathogenic mycobacteria. Mol Microbiol 2006, 62(3):667-679.

34. Gaballa A, Antelmann H, Aguilar C, Khakh SK, Song KB, Smaldone GT, Helmann JD: The Bacillus subtilis iron-sparing response is mediated by a Fur-regulated small RNA and three small, basic proteins. Proc Natl Acad SCi USA 2008.

35. Jacques JF, Jang $\mathrm{S}$, Prevost $\mathrm{K}$, Desnoyers $\mathrm{G}$, Desmarais M, Imlay J, Masse E: RyhB small RNA modulates the free intracellular iron pool and is essential for normal growth during iron limitation in Escherichia coli. Mol Microbiol 2006, 62(4):1181-1190

36. Masse $E$, Gottesman S: A small RNA regulates the expression of genes involved in iron metabolism in Escherichia coli. Proc Natl Acad Sci USA 2002, 99(7):4620-4625.

37. Bannantine JP, Huntley JF, Miltner E, Stabel JR, Bermudez LE: The Mycobacterium avium subsp. paratuberculosis $35 \mathrm{kDa}$ protein plays a role in invasion of bovine epithelial cells. Microbiology 2003, 149(Pt 8):2061-2069.

38. Bannantine JP, Radosevich TJ, Stabel JR, Berger S, Griffin JF, Paustian ML: Production and characterization of monoclonal antibodies against a major membrane protein of Mycobacterium avium subsp. paratuberculosis. Clin Vaccine Immunol 2007, 14(3):312-317.

39. Merkal RS, Curran BJ: Growth and metabolic characteristics of Mycobacterium paratuberculosis. Appl Microbiol 1974, 28(2):276-279.

40. Motiwala AS, Li L, Kapur V, Sreevatsan S: Current understanding of the genetic diversity of Mycobacterium avium subsp. paratuberculosis. Microbes Infect 2006, 8(5):1406-1418.

41. Harris NB, Robbe-Austerman S, Payeur JB: Effect of egg yolk on the detection of Mycobacterium avium subsp. paratuberculosis using the ESP II liquid culture system. J Vet Diagn Invest 2005, 17(6):554-560.
42. Whittington RJ, Sergeant ES: Progress towards understanding the spread, detection and control of Mycobacterium avium subsp paratuberculosis in animal populations. Aust Vet J 2001, 79(4):267-278.

43. Wandersman C, Delepelaire P: Bacterial iron sources: from siderophores to hemophores. Annu Rev Microbiol 2004, 58:611-647.

44. Masse E, Salvail H, Desnoyers G, Arguin M: Small RNAs controlling iron metabolism. Curr Opin Microbiol 2007, 10(2):140-145.

45. Runyen-Janecky L, Daugherty A, Lloyd B, Wellington C, Eskandarian H, Sagransky M: Role and regulation of iron-sulfur cluster biosynthesis genes in Shigella flexneri virulence. Infect Immun 2008, 76(3):1083-1092.

46. Fontecave M, Choudens SO, Py B, Barras F: Mechanisms of iron-sulfur cluster assembly: the SUF machinery. J Biol Inorg Chem 2005, 10(7):713-721.

47. Huet $G$, Daffe M, Saves I: Identification of the Mycobacterium tuberculosis SUF machinery as the exclusive mycobacterial system of [Fe-S] cluster assembly: evidence for its implication in the pathogen's survival. J Bacteriol 2005, 187(17):6137-6146

48. Savvi S, Warner DF, Kana BD, McKinney JD, Mizrahi V, Dawes SS: Functional characterization of a vitamin B12-dependent methylmalonyl pathway in Mycobacterium tuberculosis: implications for propionate metabolism during growth on fatty acids. J Bacterio/ 2008, 190(11):3886-3895.

49. Eoh H, Brown AC, Buetow L, Hunter WN, Parish T, Kaur D, Brennan PJ, Crick DC: Characterization of the Mycobacterium tuberculosis 4diphosphocytidyl-2-C-methyl-D-erythritol synthase: potential for drug development. J Bacteriol 2007, 189(24):8922-8927.

50. Miallau L, Faller M, Chiang J, Arbing M, Guo F, Cascio D, Eisenberg D: Structure and proposed activity of a member of the $\operatorname{VapBC}$ family of toxin-antitoxin systems. VapBC-5 from Mycobacterium tuberculosis. J Biol Chem 2009, 284(1):276-283.

51. Stallings CL, Stephanou NC, Chu L, Hochschild A, Nickels BE, Glickman MS: $\mathrm{CarD}$ is an essential regulator of rRNA transcription required for Mycobacterium tuberculosis persistence. Cell 2009, 138(1):146-159.

52. Lella RK, Sharma C: Eis (enhanced intracellular survival) protein of Mycobacterium tuberculosis disturbs the cross regulation of T-cells. $J$ Biol Chem 2007, 282(26):18671-18675.

53. Frankenberg-Dinkel N: Bacterial heme oxygenases. Antioxid Redox Signal 2004, 6(5):825-834

54. Chim N, Iniquez A, Nguyen TQ, Goulding CW: Unusual diheme conformation of the heme-degrading protein from Mycobacterium tuberculosis. J Mol Biol 395(3):595-608.

55. Boughammoura A, Matzanke BF, Bottger L, Reverchon $S$, Lesuisse E, Expert $D$, Franza $T$ : Differential role of ferritins in iron metabolism and virulence of the plant-pathogenic bacterium Erwinia chrysanthemi 3937. J Bacteriol 2008, 190(5):1518-1530

doi:10.1186/1471-2180-10-268

Cite this article as: Janagama et al:: Iron-sparing Response of

Mycobacterium avium subsp. paratuberculosis is strain dependent. BMC Microbiology 2010 10:268.

\section{Submit your next manuscript to BioMed Central and take full advantage of:}

- Convenient online submission

- Thorough peer review

- No space constraints or color figure charges

- Immediate publication on acceptance

- Inclusion in PubMed, CAS, Scopus and Google Scholar

- Research which is freely available for redistribution

Submit your manuscript at www.biomedcentral.com/submit
C Biomed Central 